\title{
Epidemiology of ambulance utilized patients in Addis Ababa, Ethiopia
}

Menbeu Sultan ${ }^{1 *}$, Yonas Abebe ${ }^{2}$, Assefu Welde Tsadik ${ }^{3}$, Catherine Ann Jennings ${ }^{4}$ and Nee-Kofi Mould-Millman ${ }^{5}$

\begin{abstract}
Background: Well organized and appropriately utilized pre-hospital emergency services play a critical role in augmenting emergency care systems. The primary objective of this study was to understand the demographic and clinical profile of patients who used ambulances in Addis Ababa. The secondary objectives were to assess ambulance response time, transport time and reasons for referral amongst inter-facility transported patients in Addis Ababa.

Methods: The study was designed as a cross-sectional retrospective chart review of ambulance transported patients using ambulance station records from Addis Ababa Fire and Emergency Prevention and Control Authority. With IRB approval, simple random sampling and manual review of six months of clinical records was performed. Data were collected by trained data collectors and descriptive analysis was done using SPSS version 20.

Results: Female patients used ambulance services more often than males (female to male ratio of 3:1) and the mean age of the patients was 26 years. The most commonly transported age group was 16-30 years, followed by 31-50 years and neonatal patients (i.e. $<1$ month). The majority of the patients had pregnancy related illnesses $(n=492,61.4 \%)$, followed by general medical issues $(n=210,26.2 \%)$ and injury secondary to trauma $(n=99,12.3 \%)$. Most patients $(n=702,87.6 \%)$ were transported for inter-facility transfers, while only $12.4 \%(n=99)$ were primary responses (i.e. from the scene). Prolonged labor was the most common reason $(n=103,23.4 \%)$ for inter-facility transfer of pregnant patients, followed by premature rupture of the amniotic membrane $(n=60,13.6 \%)$. The mean dispatch to scene time interval was $10.1 \mathrm{~min}$, and mean scene to facility time interval was $17.2 \mathrm{~min}$.

Conclusion: Inter-facility transfers accounted for the largest proportion of ambulance utilization and dispatch in Addis Ababa. Ambulance transport time was twice as long compared to international recommendations of less than eight minutes for emergent transports. The most common reasons for ambulance dispatch were Obstetric. We recommend urgent action to decrease the transport times and to dedicate further pre-hospital resources to address the high burden of inter-facility transfers.
\end{abstract}

Keywords: Ambulance, Epidemiology, Inter-facility transfer

\section{Background}

Emergency Medical Service (EMS) systems exist to reduce death and disability for those experiencing life or limb threatening medical emergencies in the pre-hospital environment. A primary operational task of EMS systems is to provide out of hospital medical care and transport to the most appropriate health facility for further care. A meta-analysis by Jaymie et. al in developing nations has shown a $25 \%$ decreased risk of death from trauma in areas that had a pre-hospital trauma system [1]. Furthermore, if

\footnotetext{
* Correspondence: smenbeu@yahoo.com

${ }^{1}$ Department of Emergency Medicine and Critical Care, St. Paul's Hospital

Millennium Medical College, Addis Ababa, Ethiopia

Full list of author information is available at the end of the article
}

appropriately used, ambulances are highly cost effective in reducing mortality from emergency conditions $[2,3]$.

Given the epidemiological shift from communicable diseases to non-communicable diseases occurring in Ethiopia, there is an increasing demand for EMS systems [4]. In one community-based study done from 2006 to 2009 in Addis Ababa, cardiovascular diseases accounted for $24 \%$ of deaths. Although there has been a significant reduction in pregnancy related mortality, non-communicable diseases like heart disease, stroke, and respiratory disease are increasing $[4,5]$. In postmortem examination of sudden deaths in Addis Ababa, 52\% of the individuals died from natural causes and $48 \%$ died from injury-related events. In

(c) The Author(s). 2018 Open Access This article is distributed under the terms of the Creative Commons Attribution 4.0 International License (http://creativecommons.org/licenses/by/4.0/), which permits unrestricted use, distribution, and reproduction in any medium, provided you give appropriate credit to the original author(s) and the source, provide a link to the Creative Commons license, and indicate if changes were made. The Creative Commons Public Domain Dedication waiver (http://creativecommons.org/publicdomain/zero/1.0/) applies to the data made available in this article, unless otherwise stated. 
the injury related group, road traffic injury (RTI) was the most common cause of accidental death (80\%), and no pre-hospital care was received $[4,6]$. Road traffic injury is steadily increasing and is disproportionately affecting the economically active groups (i.e. those 15-59 years of age) [7-9].

Response time, availability and training of pre-hospital providers, available resources, and the appropriateness of calls contribute to the overall quality of pre-hospital care. A common benchmark response time in high-income settings has been recommended to be less than eight minutes [10], but no evidence based equivalent exists for low and middle income settings. Addis Ababa ambulance service has a fragmented organizational structure with very limited number of ambulance vehicles [11]. Similar to reports from other African countries, in Addis Ababa the use of private cars and taxis is the current norm for transporting an emergency patient from scene to health facility [12].

In past 10 years, the Federal Ministry of Health $(\mathrm{FMoH})$ has introduced an effort to improve the EMS systems in Ethiopia. Efforts include distribution of ambulances to all regions, providing at least one ambulance per district (woreda), training of paramedics, and procurement of on-board medical equipment [13]. Addis Ababa Fire and Emergency Prevention and Control Authority (AAFEPCA), along with few private companies, provide the major pre-hospital emergency services care for the city. Under their authority, there is one central dispatch center for fire and pre-hospital services, eight ambulance stations, and about 32 ambulances.The authority provides free pre-hospital care, including scene to health facility and inter-facility transfers. A free ambulance phone number (939) is used for access by the public. The care providers are nurses with short term pre-hospital patient care training. Although the pre-hospital service has existed for over 10-years, the epidemiology of patients using ambulances and the appropriateness of this usage remains unstudied. This study aims to examine patients' clinical characteristics and reasons for ambulance use in Addis Ababa.

\section{Objective}

The primary objective of this study was to assess the demographics and clinical profiles of patients utilizing ambulances in Addis Ababa. The secondary objectives of the study were to assess ambulance response time, transport time, and reasons for referral amongst inter-facility transported patients in Addis Ababa.

\section{Methods}

\section{Study setting and design}

This study was conducted in Addis Ababa, the capital city of Ethiopia. The city has a population of more than four million people across ten sub-cities and is a site for diplomats and international organizations including the African Union and the Economic Commission for Africa. Nearly $70 \%$ of the population is younger than 30 years of age, and there is a slightly higher number of females (F:M 1.03:1). Addis Ababa Fire and Emergency Prevention and Control Authority is a government funded organization delivering free emergency and ambulance services for the public. At the time of the study, it had eight ambulance stations distributed indifferent sub-cities of the town. A cross-sectional retrospective chart review of patient records was conducted from the AAFEPCA ambulance stations.

\section{Sample size and sampling}

Four ambulance stations were randomly chosen using computer generated numbers from the available eight stations. The selected ambulance stations were Addis Ketema, Bole, Kirkos and Nifas Silk-Lafto. Each ambulance station maintained written records of ambulance transported patients. Based on data from a similar study done in Uganda, it was anticipated that $66 \%$ of ambulance utilization was for pregnancy related conditions [14], and sample size was calculated using the single proportion $P=0.66$ (where $\mathrm{P}$ is the proportion of patients with pregnancy related conditions), with $95 \%$ confidence interval, $4 \%$ of degree of freedom, and $5 \%$ added for exclusion of incomplete data. The calculated sample size of 565 was multiplied by 1.5 for the cluster effect to obtain the final sample size estimate of 847. Patient charts were selected from paper based patient registry (at the individual ambulance stations) using simple random sampling.

\section{Data collection and analysis}

The data abstraction format was prepared and pre-tested. Once patient charts were identified, study investigators screened the charts for completeness. Data were collected by data collectors trained by study investigators and the data quality was controlled by the investigators throughout the data collection time by doing double chart reviews. Data cleaning and analysis for descriptive statistics was done using SPSS version 20. Missing data were interpreted as it was without replacement.

\section{Ethical considerations}

The study protocol was reviewed and approved by St. Paul Hospital Millennium Medical College Institutional Review Board (IRB). Confidentiality was maintained throughout the study.

\section{Results}

In a six-month period from July to December of 2016, a total of 7800 patients' clinical reports existed from the four ambulance stations tested. Of those, 847 patient 
records were sampled and 46 records were then excluded due to incomplete data. Of the final 801 study participants, $(n=605,75.5 \%)$ were female, and patients aged $16-$ 30 years were the most common age group represented $(n=583,72.7 \%)$. The most common location of patients for initiation of ambulance transport was a health facility $(n=702,87.6 \%)$ and public hospitals were the most common destination facility $(n=726,90.6 \%)$. The mean ambulance response time interval was $10.1 \mathrm{~min}$ and the mean patient transport time interval was 17.2 min (Table 1).

\section{Clinical characteristics of the patients}

The majority of patients had pregnancy related illnesses $(n=492,61.4 \%)$, followed by medical illness such as respiratory distress $(n=210,26.2 \%)$ and trauma $(n=99$, $12 \%)$. On primary assessment of the patients, eight (1\%) patients had airway problems, 16(2\%) patients had breathing problems, and 77(9.8\%) of patients had severe bleeding. On the assessment of mental status, the majority of the patients were fully alert, $(n=651,81.6 \%)$. Regarding vital signs, $82(13.1 \%)$ patients had hypotension and 125(18.6\%) patients had tachypnea. Intravenous fluid administration was the most common on-board treatment given $(n=311,39 \%)$ (Table 2).

\section{Inter facility transfer of patients}

Of all study participants ambulance transportation, the majority were inter-facility transfers $(n=702,87.6 \%)$.

Table 1 Characteristics of ambulance used patients in Addis Ababa stratified by type of illness

\begin{tabular}{|c|c|c|c|c|c|c|c|}
\hline Patient characteristic & $\begin{array}{l}\text { Number of patients } \\
\text { with injury }\end{array}$ & Percentage & $\begin{array}{l}\text { Number of patients } \\
\text { with disease }\end{array}$ & Percentage & $\begin{array}{l}\text { Number of patients with } \\
\text { pregnancy related condition }\end{array}$ & Percentage & Total \\
\hline \multicolumn{8}{|c|}{ Age of the patients in years } \\
\hline $0-1$ & 0 & 0 & 32 & 100 & NA & NA & 32 \\
\hline $2-5$ & 2 & 12.5 & 14 & 87.5 & NA & NA & 16 \\
\hline $6-15$ & 5 & 31.3 & 11 & 68.8 & NA & NA & 16 \\
\hline $16-30$ & 62 & 10.6 & 88 & 15.1 & 433 & 74.3 & 583 \\
\hline $31-50$ & 22 & 18.6 & 37 & 31.4 & 59 & 50.0 & 118 \\
\hline$>50$ & 8 & 22.2 & 28 & 77.8 & 0 & .0 & 36 \\
\hline \multicolumn{8}{|l|}{ Sex } \\
\hline Male & 77 & 39.3 & 119 & 60.7 & NA & NA & 196 \\
\hline Female & 22 & 3.6 & 91 & 15.0 & 492 & 81.3 & 605 \\
\hline \multicolumn{8}{|l|}{ Scene location } \\
\hline Health facility & 80 & 11.4 & 176 & 25.1 & 446 & 63.5 & 702 \\
\hline Home & 3 & 5.3 & 11 & 19.3 & 43 & 75.4 & 57 \\
\hline Industry & 1 & 11.1 & 7 & 77.8 & 1 & 11.1 & 9 \\
\hline Road & 13 & 46.4 & 14 & 50.0 & 1 & 3.6 & 28 \\
\hline Work place & 0 & 0 & 2 & 66.7 & 1 & 33.3 & 3 \\
\hline Other & 2 & 100 & 0 & .0 & 0 & 0.0 & 2 \\
\hline \multicolumn{8}{|c|}{ Minutesfrom dispatch to scene location } \\
\hline $0-9$ & 57 & 11.7 & 139 & 28.5 & 291 & 59.8 & 487 \\
\hline $10-19$ & 23 & 10.3 & 48 & 21.6 & 151 & 68.0 & 222 \\
\hline $20-39$ & 16 & 18.8 & 23 & 27.1 & 46 & 54.1 & 85 \\
\hline$>-40$ & 3 & 42.8 & 0 & 0.0 & 4 & 57.1 & 7 \\
\hline \multicolumn{8}{|c|}{ Minutes from scene to destination } \\
\hline $0-9$ & 13 & 7.5 & 51 & 29.3 & 110 & 63.2 & 174 \\
\hline $10-19$ & 40 & 12.8 & 61 & 19.6 & 211 & 67.63 & 312 \\
\hline 20-39 & 39 & 15.5 & 73 & 28.9 & 140 & 55.6 & 252 \\
\hline$>40$ & 6 & 22.2 & 13 & 48.2 & 8 & 29.6 & 27 \\
\hline \multicolumn{8}{|l|}{ Receiving health institute } \\
\hline Public hospital & 84 & 11.6 & 184 & 25.3 & 458 & 63.1 & 726 \\
\hline Public health center & 15 & 20.0 & 26 & 34.7 & 34 & 45.3 & 75 \\
\hline
\end{tabular}


Table 2 Clinical data of ambulance transported patients in Addis Ababa, Ethiopia

\begin{tabular}{|c|c|c|c|}
\hline & Parameter & $\begin{array}{l}\text { Number of patients } \\
\text { with condition }\end{array}$ & Percentage \\
\hline \multirow[t]{2}{*}{ Air way $(n=798)$} & Clear & 790 & 99.0 \\
\hline & Partly blocked & 8 & 1.00 \\
\hline \multirow[t]{2}{*}{ Breathing $(n=798)$} & Effective & 782 & 98.0 \\
\hline & Ineffective & 16 & 2.00 \\
\hline \multirow[t]{2}{*}{ Severe bleeding $(n=798)$} & Yes & 77 & 9.60 \\
\hline & No & 721 & 90.4 \\
\hline \multirow[t]{3}{*}{ Respiratory rate for age $(n=673)$} & Tachypnea & 125 & 18.7 \\
\hline & Normal & 537 & 79.8 \\
\hline & Bradypnea & 11 & 1.6 \\
\hline \multirow[t]{3}{*}{ Pulse rate for age $(n=711)$} & Fast & 46 & 6.5 \\
\hline & Normal & 651 & 91.6 \\
\hline & Slow & 14 & 1.90 \\
\hline \multirow[t]{2}{*}{ Blood pressure $(n=616)$} & Normal & 534 & 86.7 \\
\hline & Low & 82 & 13.3 \\
\hline \multirow[t]{4}{*}{ Casualty responsiveness $(n=798)$} & Alert & 651 & 81.6 \\
\hline & Verbal response & 112 & 14.0 \\
\hline & Responds to pain & 22 & 2.76 \\
\hline & Unresponsive & 13 & 1.6 \\
\hline \multirow{5}{*}{$\begin{array}{l}\text { Treatment given in the } \\
\text { ambulance }(n=485)\end{array}$} & IV line & 311 & 64.1 \\
\hline & Oxygen & 107 & 22.1 \\
\hline & Bleeding Arrest & 62 & 12.8 \\
\hline & Deliver care & 3 & 0.6 \\
\hline & CPR & 2 & 0.4 \\
\hline
\end{tabular}

Key: ${ }^{2}$ Low blood pressure for adults $<90 / 60 \mathrm{mmgh}$, Normal systolic $90-120 \mathrm{mmgh}$.fast pulse rates for adults $>110$ per minute, Brady cardia is heart rate less than 60 per minute, tachypnea is RR $>22$ per minute for adults

Health centers (the lowest level of public health facility in Addis Ababa) were the most common referring health institutions, accounting for $82.6 \%$ of cases $(n=578)$, followed by public hospitals ( $n=103,14.7 \%)$. Pregnancy related causes were the most common reasons for inter facility transfer $(N=446,63.5 \%)$. Prolonged labor $(n=103$, $24.0 \%$ ) was the most commonly stated reason for pregnancy related inter-facility transfer, followed by premature rupture of amniotic membrane (PROM) $(n=60,14.0 \%)$ (Fig. 1). Non-pregnancy related causes (i.e. trauma and medical) of inter-facility transfer account for $36.5 \%$ of cases $(n=256)$. Of these non-obstetric inter-facility transfer cases, trauma accounted for $30 \%(n=80)$, general surgical patients for $7.8 \%(n=20)$, sick newborns for $8.8 \%(n=23)$, and the remaining $52.0 \%(n=133)$ had medical illness. Figure 2 details common medical illness for inter-facility transfer.

\section{Discussion}

During the surveyed time period, pregnancy related emergencies were the most common reasons for ambulance use in Addis Ababa (61.4\%). Additionally, the majority of ambulance use was to conduct inter-facility transfer of patients. On primary survey, severe bleeding was found as the most common problem accounting for 77 cases $(9.6 \%)$, followed by breathing problems. The most common non-obstetric referral was injury, which accounted for about one-third of all transports. A prior study in Addis Ababa identified that $41.2 \%$ of the injuries occurred on the road and $12.0 \%$ at home [6]. The majority $(81.4 \%)$ of the patients did not receive post-crash care at the pre-hospital level and $41.2 \%$ died outside the hospitals $[6,15]$. Moreover, our finding showed ambulances are mainly being used for inter-facility transfer of patients; these findings show indicate there should be an action to shift the service focus of ambulances to conduct more primary (scene) responses.

In a similar report from the Ashanti Region in Ghana, $76 \%$ of all national cases were inter-facility transports [12]. A study by Stewart de Ramirez et al. in Uganda showed that $66 \%$ of inter-facility transfers were obstetric related and $77.8 \%$ were for female patients [14]. Furthermore, $52 \%$ of all obstetric transfers and $62 \%$ of all trauma transfers were from low level to high level 


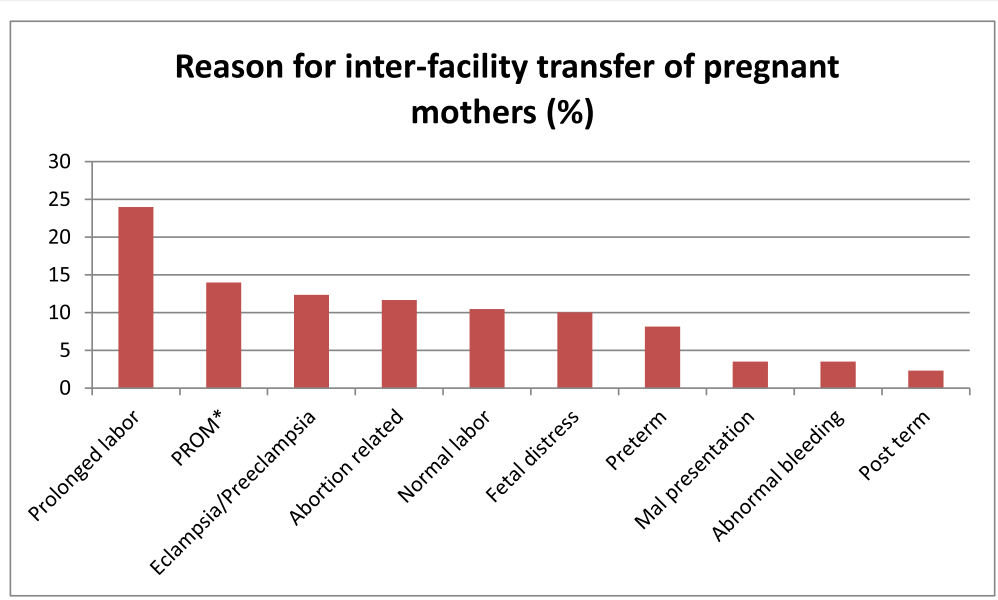

Key: * Premature Rupture of the Membrane

Fig. 1 Common pregnancy related illnesses requiring inter facility ambulance transportation in Addis Ababa

hospital care [14]. These African studies, including this study in Addis Ababa, show a high burden of ambulance use for inter-facility transfers [16]. In addition, there are reports of secondary over-triage, including the unnecessary referral of patients with mild conditions to a higher level of care facility, leading to potentially un-necessary transfer of patients. A recent study done in the KwazuluNatal district of South Africa reported an in appropriate dispatch of resource, where more than $58 \%$ of cases required either no intervention or transport only [17, 18]. Our study results may indicate the necessity for increasing the capacity of the health centers and providers to prevent unnecessary inter-facility transfers in the study settings.

Amongst pregnancy related transfers, prolonged labor was the most commonly stated reason for transfer $(n=103$, $24.0 \%)$, followed by premature rupture of amniotic membrane $(n=60,14.0 \%)$. Additionally, postpartum hemorrhage accounted for 15 cases (4\%) of obstetric transfers and neonatal emergencies accounted for 23 cases $(8.8 \%)$ of non-obstetrictransfers. In a 2013 study of Addis Ababa health centers, poor provider competency was reported, with insufficient knowledge for diagnosing postpartum hemorrhage (PPH) and birth asphyxia, as well as poor skills in neonatal resuscitation [19]. Given that the majority of transfers were from health centers to hospitals, there may be a need for capacity building in the health centers to decrease the need for referrals. In addition, unnecessary transfer of patients, like those who have PROM, can be reduced by implementing an obstetric ambulance referral protocol and improving training of health care providers in health centers.

Most ambulance used patients are pregnant mothers having prolonged labor when time is crucial to decrease both maternal and fetal morbidity and mortality. The response time for ambulances is an important quality of ambulance care for time sensitive emergency conditions. Results identified that the ambulances in Addis Ababa are spending more than nine minutes to reach the scene.

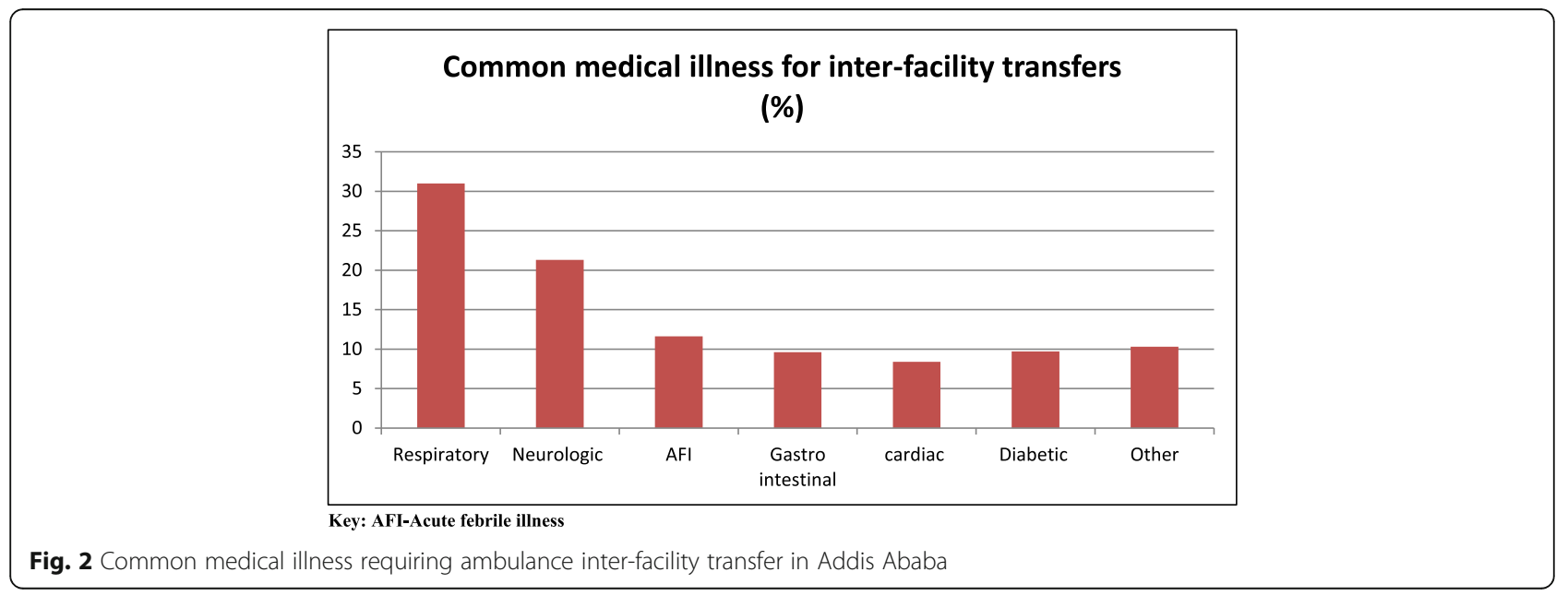


However, research shows the mortality risk for patients with ambulance response time of more than five minutes is three times higher than those less than five minutes [20]. The estimated effect of a one minute reduction in response time shows improved odds of survival by $24 \%$ [21].

One method for decreasing response time is increasing the number of available ambulances, although in one South African study, only increasing the number of ambulances did not help meet the urban priority response time goals [22]. In another South African study, emergency ambulance location had a greater effect on the response time performance [23]. Yet another study showed that increasing the ambulance base and using satellite deployment with variations according to demand and time of the day significantly decreased the median monthly response time and the proportion of patients transported in less than eight minutes [24].

There were eight ambulance stations in Addis Ababa at the time of this study. In addition to improving the road for ambulances, the base and distribution of the ambulances should also be evaluated and improved to decrease the response time. For example, dynamic postings, increasing the number of ambulances, and allocating ambulances to prioritize inter-facility transfers versus primary/scene responses can improve the disproportionate utilization of ambulance for inter-facility transfer.

\section{Limitations of the study}

The findings and interpretations are limited by the retrospective nature of the chart review and instances of missing data. The study involved only the capital city which makes findings and recommendations difficult to generalize to other non-urban metropolitan areas in Ethiopia.

\section{Conclusion}

Inter-facility transfer of patients account for the predominant proportion of ambulance dispatches in Addis Ababa. Ambulances had relatively prolonged response and transport time intervals compared to international high-priority benchmarks. The common causes for ambulance dispatch were pregnancy related emergencies. Action is needed to decrease the transport times and to dedicate further resources to address the high burden of inter-facility transfers.

\section{Abbreviations \\ AAFEPCA: Addis Ababa fire emergency prevention and control authority; ED: Emergency department; EMS: Emergency medical services; RTI: Road traffic injury; SPSS: Statistical package for the social sciences}

\section{Acknowledgements}

Authors are grateful to Addis Ababa Fire Emergency Prevention and Control Authority administration for facilitating data collection.

\section{Funding}

No funding was received for this work.
Availability of data and materials

The datasets for this work is available from the corresponding author on reasonable request.

\section{Authors' contributions}

MS, YA, AW, CJ and NM designed the study. MS and YAtrained the data collectors, monitored the data collection and analyzed the data. All authors participated in data interpretation.MS wrote the manuscript. All authors contributed critical revisions to the report, and all approved the final version.

\section{Ethics approval and consent to participate}

The study protocol was approved by the Institutional Review Board of St. Paul's Hospital Millennium Medical College.

\section{Consent for publication}

Not applicable.

\section{Competing interests}

The authors declare that they have no competing interests.

\section{Publisher's Note}

Springer Nature remains neutral with regard to jurisdictional claims in published maps and institutional affiliations.

\section{Author details}

'Department of Emergency Medicine and Critical Care, St. Paul's Hospital Millennium Medical College, Addis Ababa, Ethiopia. ²Department of Emergency Medicine and Critical Care Nursing, St. Paul's Hospital Millennium Medical College, Addis Ababa, Ethiopia. ${ }^{3}$ Federal Ministry of Health Ethiopia, Emergency and Critical Care Directorate, Addis Ababa, Ethiopia. ${ }^{4}$ Columbia University Premedical Post baccalaureate, New York, USA. ${ }^{5}$ University of Colorado School of Medicine, Aurora, USA

Received: 6 August 2018 Accepted: 16 December 2018

Published online: 27 December 2018

References

1. Ang J, Arthur H, Reingold L. Prehospital trauma systems reduce mortality in developing countries: a systematic review and meta-analysis. J Trauma Acute Care Surg. 2012;73(1):261-8.

2. Godefay H, Kinsman J, Admasu K, Byass P. Can innovative ambulance transport avert pregnancy-related deaths? One-year operational assessment in Ethiopia. J Glob Health. 2016;6(1):1-9.

3. Accorsi S, Somigliana E, Solomon H, Ademe T, et al. Cost-effectiveness of an ambulance-based referral system for emergency obstetrical and neonatal care in rural Ethiopia. BMC Pregnancy Childbirth. 2017;17(220):1-7.

4. Misganaw A, Mariam DH, Araya T, Ayele K. Patterns of mortality in public and private hospitals of Addis Ababa, Ethiopia. BMC Public Health. 2012;12:1007.

5. Misganaw A, Mariam DH, Araya T. The double mortality burden among adults in Addis Ababa, Ethiopia, 2006-2009. Prev Chronic. 2012;9:11-0142.

6. Tufa Gemechu, Mihrete Tinsae, Senait Ashenafi, Victor Manuel Rodriguez, Alfredo Lori, Michelle Collins. Most common causes of natural and injuryrelated deaths in Addis Ababa, Ethiopia. NIH Public Access Author Manuscr 2009:205: 608-14;

7. Azaj A, Seyoum N, Nega B. Trauma in Ethiopia revisited: a systematic review. East Cent Afr J Surg. 2013;18(2):108-18.

8. Fekede Asefa, Demeke Assefa, Gezahegn Tesfaye. Magnitude of, trends in, and associated factors of road traffic collision in central Ethiopia. BMC Public Health. 2014;14:1072

9. TeferiAbegaz YB, Worku A, Assrat A, Assefa A. Road traffic deaths and injuries are under-reported in Ethiopia: a capture-recapture method. PLoS One. 2014;9(7):1-5.

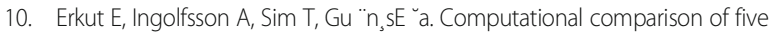
maximal covering models for locating ambulances. Geogr Anal. 2009;41:43-65

11. Germ F, Bayleyegn T, Kebede T, Ducharme J, Bartolomeos K. Emergency medicine development in Ethiopia: challenges, progress and possibilities. Afr J Emerg Med. 2013;3:3-9.

12. Mould-Millman N-C, Romiski S, Oteng R. Ambulance or taxi: high acuity prehospital transport in the Ashanti regions of Ghana. Afr J Emerg Med. 2014;4:8-13. 
13. Menbeu Sultan, Finot Debebe, Aklilu Azazh. The Status of Emergency Medicine in Ethiopia. Challenges and opportunities. Ethiop Med J. 2018:56(2):119-24.

14. de Ramirez SS, Doll J, Carle S, Anest T. Emergency Response in Resourcepoor Settings: A review of a newly-implemented EMS system in rural Uganda. PublOnline. 2014;29(3):311-6.

15. Tsegaye F, Abdella K, Ahmed E, Tadesse T, Bartolomeos K. Pattern of fatal injuries in Addis Ababa. Ethiopia: A One-year Audit. 2010;15(2):10-7.

16. Mould-Millman NK, Dixon JM, Sefa N, Yancey A, Hollong BG, Hagahmed M, Ginde AA, Wallis LA. The state of Emergency Medical Services (EMS) systems in Africa. Prehosp Disaster Med. 2017;32(3):1-11.

17. Newton PR, Naidoo R, Brysiewicz P. The appropriateness of emergency medical service responses in the Thekwini district of KwaZulu-Natal, South Africa. SAMJ. 2015;105(10):844-7.

18. Meredith J. Sorensen, Friedrich M von Recklinghausen, Gwendolyn Fulton, Kenneth W. Burchard,. Secondary Overtriage the burden of unnecessary Interfacility transfers in a RuralTraumaSystem. JAMA Surg 2013;148(8):763-768.

19. Mirkuzie AH, Sisay MM, Reta AT, Bedane MM. Current evidence on basic emergency obstetric and newborn care services in Addis Ababa, Ethiopia; a cross sectional study. BMC Pregnancy Childbirth. 2014;354(14):2-8.

20. Blackwell TH, Kaufman JS. Responce time effectiveness: comparison of response time and survival in an urban emergency medical services system. Acad Emerg Med. 2002;9(4):288-95.

21. O'Keeffe C, Turner JNJ, Goodacre S. Role of ambulance response times in the survival of patients with out-of-hospital cardiac arrest. Emerg Med J. 2011;28(8):703-6.

22. Stein $C$, Wallis $L$, Adetunji $O$. Meeting national response time targets for priority lincidents in anurban emergency medical services system in South Africa: more ambulances won't help. SAMJ. 2015;105(10):40-4.

23. Stein $C$, Wallis $L$, Adetunji $O$. The effect of the emergency medical services VehicleLocation and response strategy on response times. South African Journal of Industrial Engineering. 2015;26(2):26-40.

24. OM P, Chiam TF, Ng FS, Sultana P, et al. Reducing ambulance response times using geospatial-time analysis of ambulance deployment. Acad Emerg Med. 2010;17(9):951-7.

Ready to submit your research? Choose BMC and benefit from:

- fast, convenient online submission

- thorough peer review by experienced researchers in your field

- rapid publication on acceptance

- support for research data, including large and complex data types

- gold Open Access which fosters wider collaboration and increased citations

- maximum visibility for your research: over $100 \mathrm{M}$ website views per year

At $\mathrm{BMC}$, research is always in progress.

Learn more biomedcentral.com/submissions 